\title{
Reduced expression of the gap junction protein Connexin 43 in keratoconus
}

\begin{abstract}
Purpose The purpose of the present study was to examine whether keratoconus, which is a bilateral noninflammatory corneal ectasia with multifactorial aetiology, shows altered expression of Connexin (Cx43). Cx43 is an important gap junction protein that contributes crucially to epithelial and stromal integrity of cornea.

Methods Eight keratoconic human corneal buttons were examined with immunohistochemistry and Western blotting and compared with eight normal human corneal buttons, to unravel changes in $\mathrm{Cx} 43$ expression.
\end{abstract}

Results All normal corneas exhibited similar epithelial Cx43 expression patterns, with the protein located in the basal epithelial layer. In contrast, some keratoconic corneas showed an altered pattern of immunostaining and Western blotting confirmed a decreased expression of $\mathrm{Cx} 43$ in keratoconic corneas. Conclusions Our results indicate that a decrease in Cx43 amount together with

Department of Experimental Ophthalmology, University Eye Hospital Muenster, Muenster, Germany

Correspondence: S Thanos, Department of Experimental Ophthalmology,

School of Medicine, University of Münster, Domagkstraße 15, Münster 48149,

Germany

Tel.: + 492518356915 ;

Fax + 492518356916

E-mail: solon@

uni-muenster.de

Received: 24 October 2006 Accepted in revised form: 3 August 2007

Published online:

14 September 2007 functional alteration of the protein is associated with keratoconus pathophysiology However, these changes apply only to some of the corneas examined and may not generally account for the development of keratoconus. Eye (2008) 22, 294-299; doi:10.1038/sj.eye.6702972; published online 14 September 2007

Keywords: keratoconus; Connexin 43; cornea; immunostaining; western blotting

\section{Introduction}

Keratoconus is a bilateral corneal disorder with an incidence of approximately 1 per 2000 in the general population, in which the central cornea assumes a conical shape as a result of noninflammatory thinning of the corneal

\section{Z Gatzioufas, P Charalambous and S Thanos}

stroma. ${ }^{1}$ The corneal thinning induces protrusion, irregular astigmatism, and myopia, leading to impairment in the quality of vision, with the likelihood of progression to corneal transplantation ranging from 10 to $20 \%{ }^{2}$ Despite extensive basic research and clinical investigation, the pathophysiological processes underlying keratoconus are not elucidated yet. Several mechanisms are suggested to be involved in the pathophysiology of this disorder; degradation in basal corneal epithelium, and corneal stroma is the common pathogenetic pathway of damage though. ${ }^{3}$ Recent data provide strong evidence that the development of keratoconus involves a high degree of inter- and intralamellar collagen displacement and slippage induced by a loss of cohesive forces and mechanical failure. ${ }^{4}$

Corneal gap junctions are specialized cellsurface membrane channels that directly connect adjacent cells and permit the intercellular passage of small molecules up to $1 \mathrm{kDa} .{ }^{5}$ They consist of two hemichannels, also termed connexons, which are located in the plasma membrane of two adjacent cells; each connexon is composed of six protein subunits called connexins (Cxs). Gap junction intercellular communication is crucial for the regulation of cell growth, proliferation, and differentiation and thereby contributes to the maintenance of tissue homoeostasis. ${ }^{6}$

Molecular studies have revealed more than 20 mammalian members of the connexin protein family. ${ }^{7}$ Two gap junction proteins Connexin 43 (Cx43) and Connexin 50 (Cx50) have been identified in human corneal epithelium, while Cx43 gap junctions have also been detected in corneal stroma. ${ }^{8,9}$ Corneal gap junctions mediate the intercellular transfer of ions and low molecular weight metabolites in basal epithelium and stroma through a complicated phosphorylation-induced mechanism of activation and thus, determine the cell metabolic synchrony and cooperation within 
the corneal epithelium as well as the corneal tissue differentiation and homoeostasis. ${ }^{10,11}$

In the present study, we examined the expression of gap junction protein $\mathrm{C} \times 43$ in keratoconus, which is characterized by altered epithelial and stromal metabolism in the cornea, to unravel its potential role in the pathophysiology of the disease.

\section{Methods}

\section{Immunohistochemical staining}

We examined eight human corneal buttons of $7.5 \mathrm{~mm}$ diameter from normal donors (four of male origin/four of female origin) that did not fulfil the necessary criteria for transplantation and eight human buttons of $8 \mathrm{~mm}$ diameter from keratoconus patients (four of male origin/ four of female origin) who underwent penetrating keratoplasty. The mean age of the donors was 32 years (range, 25-56) and the mean age of the keratoconus patients was 48.6 years (range, 22-66). The demographic and clinical characteristics of the keratoconus patients are depicted in Table 1. All tissues were obtained from the local Cornea Bank (University Eye Hospital, Muenster, Germany). Normal and keratoconus corneas were examined immediately after excision. Human tissue was handled according to the tenets of the Declaration of Helsinki. All corneas were divided into two equal segments; each half was sectioned for immunohistochemistry, while the other half was used for Western blotting. Small tissue blocks $(2 \times 2 \mathrm{~mm})$ from the central $6 \mathrm{~mm}$ from every cornea were frozen in liquid nitrogen and then transferred to cryostat (Leika Jung CM 1500; Leica Microsystems, Wetzlar, Germany) and cut into frozen sections ( $12 \mu \mathrm{m}$ thick). Specimens were collected on clean gelatine-coated glass slides and dried for $2 \mathrm{~h}$ at room temperature. All sections were fixed in cold methanol $99 \%$ for $10^{\prime}$ in $-20^{\circ} \mathrm{C}$, washed three times for $5 \mathrm{~min}$ each in phosphate-buffered saline and blocked with $10 \%$ fetal calf serum for $30 \mathrm{~min}$.

Table 1 Demographic and clinical data of keratoconus patients

\begin{tabular}{|c|c|c|c|c|c|c|c|c|c|}
\hline$N / N$ & Race & Gender & Age & $K G$ & $C L$ & $\mathrm{CH}$ & $D S$ & $P K$ & First PK \\
\hline 1 & W & F & 66 & Moderate & No & No & No & $\mathrm{R}$ & Yes \\
\hline 2 & W & M & 63 & Severe & Yes & Yes & No & $\mathrm{R}$ & Yes \\
\hline 3 & W & M & 22 & Severe & No & No & No & $\mathrm{L}$ & Yes \\
\hline 4 & W & M & 39 & Severe & Yes & No & No & $\mathrm{R}$ & Yes \\
\hline 5 & B & F & 47 & Moderate & No & Yes & No & $\mathrm{L}$ & Yes \\
\hline 6 & W & F & 49 & Severe & Yes & No & No & L & Yes \\
\hline 7 & W & M & 44 & Severe & No & Yes & Yes & $\mathrm{R}$ & Yes \\
\hline 8 & W & $\mathrm{F}$ & 59 & Severe & Yes & No & No & $\mathrm{R}$ & Yes \\
\hline
\end{tabular}

B, black race; $\mathrm{CH}$, corneal hydrops; $\mathrm{CL}$, contact lens wear at least for one year before operation; DS, Down syndrome; F, female; KG, keratoconus grade according to the CLAO criteria; L, left eye; M, male; PK, penetrating keratoplasty; $\mathrm{R}$, right eye; $\mathrm{W}$, white race.
Immunofluorescence staining was performed using a primary antibody against $\mathrm{Cx} 43$ (affinity purified goat polyclonal antibody; Santa Cruz Biotechnology, Inc., Santa Cruz, CA, USA). Primary antibody was diluted in $10 \%$ fetal calf serum $(1: 100)$ and the sections incubated overnight at $4{ }^{\circ} \mathrm{C}$. After rinsing the slides three times for $5 \mathrm{~min}$ each in phosphate-buffered saline, the sections were incubated for $30^{\prime}$ with the secondary antibody (donkey anti-goat Cy2-conjugated antibody; Jackson ImmunoResearch Laboratories Inc., West Grove, PA, USA). Thereafter, it was once again washed three times for $5^{\prime}$ each in phosphate-buffered saline and finally mounted in anti-fading solution for fluorescence microscopy (Mowiol, Merck, Darmstadt, Germany) containing bisbenzimide H 33258 (DAPI; Sigma, St Louis, $\mathrm{MO}$, USA) and observed with the aid of a fluorescence microscope (Axiophot; Carl Zeiss Meditec, Oberkochen, Germany) using appropriate filters (395-440 nm for Dapi and 450-490 nm for Cy2). Photography was achieved digitally with a camera attached to the microscope (AxioCam, Carl Zeiss Meditec, Oberkochen, Germany). Multiple immunostaining experiments were performed on each cornea (at least four sets of independent experiments on different samples of each cornea) and the statistical comparisons were based on these results.

\section{Western blotting}

Each of the eight normal human corneal pieces and eight intact pieces from keratoconic human corneas examined immunohistochemically, were also used in Western immunoblot analysis. Corneal tissue was suspended in lysate buffer containing $10 \mathrm{mM}$ Tris- $\mathrm{Cl}$ ( $\mathrm{pH} 7.4$; SigmaAldrich, St Louis, MO, USA), 1\% Triton X-100 (SigmaAldrich, St Louis, MO, USA), $5 \mathrm{mM}$ EDTA, and $0.15 \mathrm{M}$ $\mathrm{NaCl}$. The lysate was sonicated three times for $5 \mathrm{~min}$ each to completely disrupt all cells and afterwards centrifuged at 20000 r.p.m. for $30 \mathrm{~min}$ at $4^{\circ} \mathrm{C}$. The protein concentration was determined by Bradford assay. Gel electrophoresis was performed on $60 \mu \mathrm{g}$ aliquots of each sample on a SDS(12\% polyacrylamide) gel. Proteins were then transferred onto a nitrocellulose membrane. After treating the membrane with a $5 \%$ non-fat dried milkTBST blocking solution and washing out $1 \times 15$ and $3 \times 5$ min with TBST, the first antibody was added (dilution $1: 200$ ) and the membrane was incubated overnight at $4{ }^{\circ} \mathrm{C}$. The membrane was thereafter washed out $1 \times 15 \mathrm{~min}$ and $3 \times 5 \mathrm{~min}$ and consequently incubated for $45 \mathrm{~min}$ at room temperature with the secondary antibody (dilution 1:30000). An ECL chemiluminescence kit (Amersham, Buckinghamshire, UK) was used for immunoreactive detection. After $5^{\prime} \mathrm{min}$, the membrane was exposed to X-ray film and the density bands were marked with the corresponding molecular weight. 
Protein expression values in normal and keratoconus corneas were normalized by comparison to the Cx43 expression in rat brain, which served as control tissue. Densitometry was performed with aid of the AlphaEaseFC analysis software (Alpha Innotech Corporation, CA, USA).

\section{Statistical analysis}

The paired $t$-test was used to determine whether the differences in the relative intensities of the reactive bands were statistically significant. Band intensities were normalized with the band intensity of the control tissue (rat brain). Data were collected from a minimum of four different sets of experiments and were represented as mean band intensity for phosphorylated and nonphosphorylated Cx43 ( $\mathrm{mP}$ and $\mathrm{mNP}$ respectively). Statistical analysis was carried out with SPSS statistic package (SPSS for Windows, Version 11.0, SPSS Inc., Chicago, IL, USA). A $P$-value less than 0.05 , was considered statistically significant.

\section{Results}

\section{Immunohistochemistry}

As Figure 1 shows, immunostaining showed that all normal human corneas strongly expressed Cx43 in the basal epithelial layers as well as in the anterior stroma. The signal was detected continuously along the basic membrane and sporadically in the keratocytes of the anterior stroma (Figure $1 \mathrm{~b}$ and $\mathrm{c}$ ). In the keratoconic human corneas, in four out of eight corneas (corneas 3, 4, 6, and 8) Cx43 immunolabelling was observed sporadically in the basal epithelium (Figure 1e and f), whereas two corneas showed no staining (corneas 2 and 7). In two corneas, the Cx43 expression was characterized by an immunostaining pattern similar to normal corneas (corneas 1 and 5).

\section{Western blotting}

In Western blotting analysis, four specific signals for Cx43 were detected, corresponding to molecular sizes of $43,47,48$, and $49 \mathrm{kDa}$. The $43 \mathrm{kDa}$ band represented the nonphosphorylated Cx43 (NP-Cx43), whereas the three other bands corresponded to the phosphorylated isoforms of Cx43 (P1-Cx43, P2-Cx43, and P3-Cx43 respectively).

In three keratoconus corneas (corneas 2, 6, and 7) both the NP-Cx43 and P-Cx43 isoforms were expressed less than in normal cornea (Figure 2a). The expression of NPCx43 was reduced in three keratoconus corneas (corneas 3,4 , and 8) compared to normal corneas, but not the
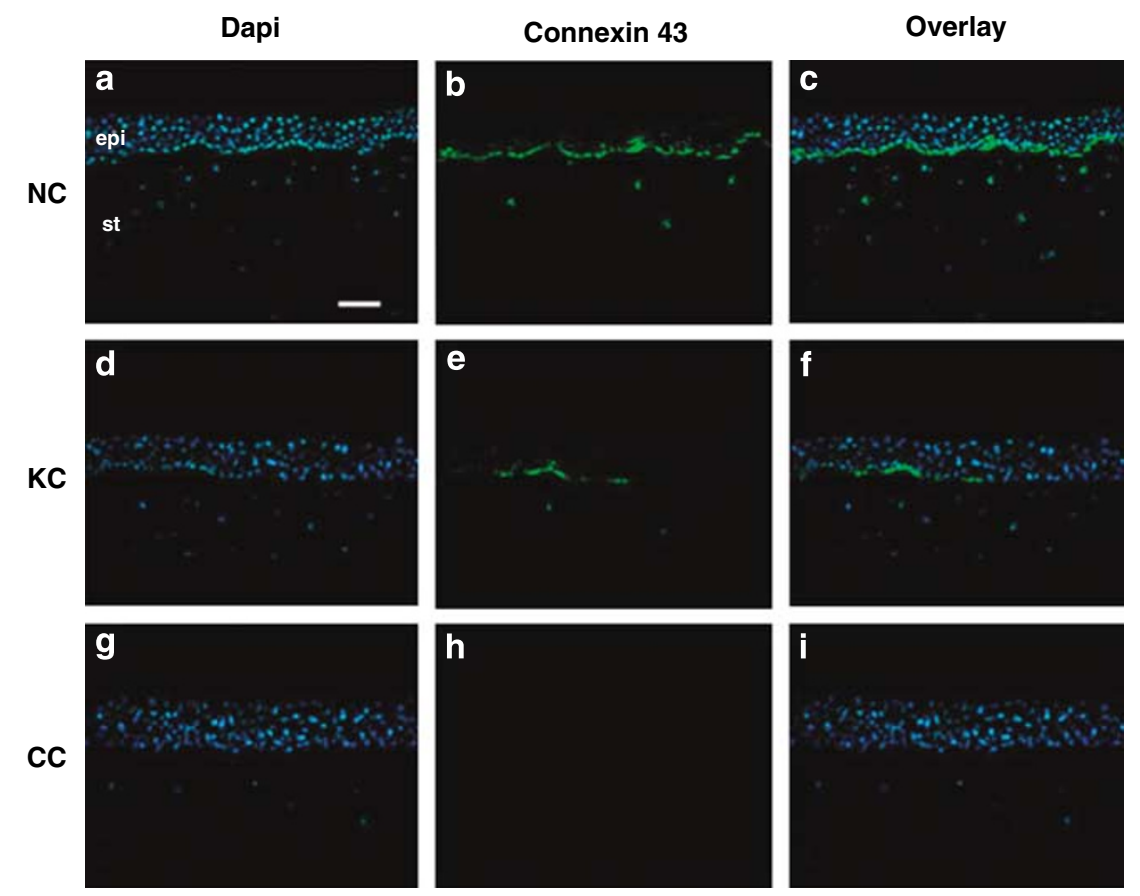

Figure 1 Immunohistochemical expression of $\mathrm{Cx} 43$ in human cornea. $\mathrm{NC}$, normal cornea; $\mathrm{KC}$, keratoconus cornea; $\mathrm{CC}$, control cornea (unstained normal cornea); epi, epithelium; st, stroma. Nuclei of corneal epithelial cells are stained blue (Dapi). Positive immunolabelling appears green (Cy2). Immunostaining for $\mathrm{C} \times 43$ was positive in basal epithelial layer of normal corneas, displaying a punctate pattern, as well as in anterior stroma ( $b$ and c). Keratoconus corneas exhibited an altered immunolabelling pattern for $\mathrm{Cx} 43$, distributed sporadically in basal epithelium and, to a smaller extent, in anterior stroma (e and f). Control corneas were not treated with the primary antibody against Cx43 and therefore showed no signal (h and i). Scale bar: $100 \mu \mathrm{m}$. 
P-Cx43 isoforms quantity (Figure 2b). Finally, in two keratoconus corneas (corneas 1 and 5) the protein expression of both NP-Cx43 and P-Cx43 were comparable to normal corneas (Figure 2c).

The mean NP-Cx43 and P-Cx43 band intensity (mNP and $\mathrm{mP}$ respectively) for the keratoconus corneas and the normal corneas were estimated by densitometric analysis. The mNP for keratoconus corneas was significantly decreased compared to normal corneas $(P<0.05)$. The $\mathrm{mP}$ was also reduced in keratoconus corneas compared to normal corneas, but the difference was not statistically significant $(P>0.05)$. The averaged values of $\mathrm{mNP}$ and $\mathrm{mP}$ for keratoconus and normal corneas are depicted in Figure 3.

\section{Discussion}

Keratoconus is a noninflammatory corneal disease with multifactorial aetiology, which is not completely understood. The stromal collagen metabolism is regulated by the proteolytic activity of matrix metalloproteinase enzymes (MMPs) and lysosomal proteinases (cathepsin G, cathepsin B) and the inhibitory

a

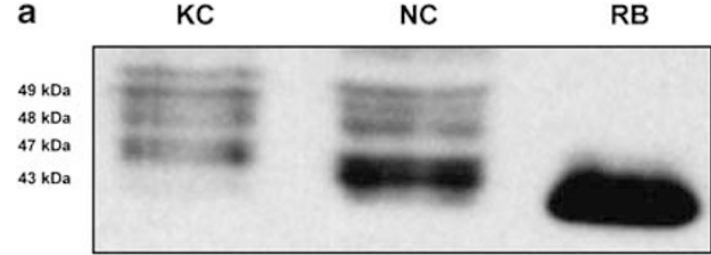

b
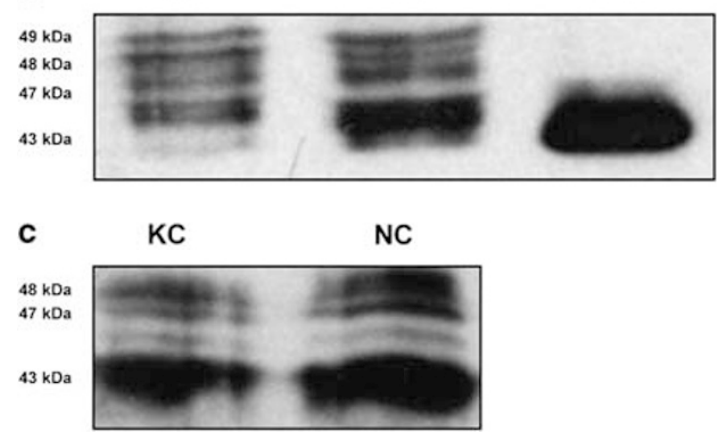

Figure 2 Protein expression of $\mathrm{Cx} 43$ in human cornea. NC normal cornea; KC, keratoconus cornea; RB, rat brain. In Western blotting analysis, four specific signals for $\mathrm{Cx} 43$ were detected, corresponding to molecular sizes of 43,47,48, and $48 \mathrm{kDa}$. The $43 \mathrm{kDa}$ band represents the nonphosphorylated Cx43 (NP-Cx43), whereas the three other bands correspond to the phosphorylated isoforms of Cx43 (P1-Cx43, P2-Cx43, and P3Cx43 respectively). Both the NP-Cx43 and $\mathrm{P}-\mathrm{C} x 43$ isoforms are expressed less in keratoconus cornea than in normal cornea (a). NP-Cx43 is decreased in keratoconus cornea compared to normal cornea, but not the P-Cx43 isoforms quantity (b). The protein expression of both NP-Cx43 and P-Cx43 in keratoconus cornea is comparable to normal cornea (c). activity of tissue inhibitors of metalloproteinases (TIMPs), a-1 proteinase inhibitor and a-2 macroglobulin. ${ }^{12}$ Keratoconic corneas exhibit increased gelatinolytic activity (MMP-2, MMP-9), as well as decreased tissue proteinase-inhibitory activity (TIMP-1, TIMP-2) with an increase in the MMP/TIMP ratio. ${ }^{13-15}$ Significant reduction of TIMP and a-2 macroglobulin levels is also reported. ${ }^{16}$ In situ hybridization and realtime PCR techniques showed that m-RNA levels of cathepsin G and cathepsin B and cathepsin V/L2 in keratoconic corneas are increased. ${ }^{17}$ In addition, immunochemistry experiments demonstrated an MT-1 MMP upregulation in keratoconic corneas and real-time PCR showed increased expression of the transcription factor Sp1, which is essential for the MT-1 MMP activation. ${ }^{18}$ It has also been proved by enzyme-linked immunoabsorbent techniques that the tear film of patients with keratoconus contains significantly higher levels of collagen degradation products (telopeptides), MMP-9, TNF-a, and IL-6 compared to normal subjects. ${ }^{19}$

Cx43 is an important gap junction protein expressed in the basal layers of the corneal epithelium and in anterior stroma. ${ }^{8,9} \mathrm{Cx} 43$ gap junctions mediate the intercellular diffusion of ions or molecules with a size of $<1 \mathrm{kDa}$ and contribute crucially to the regulation of corneal cell growth and differentiation, thereby having a significant impact on the maintenance of the corneal homoeostasis. ${ }^{20,21}$ We found that normal corneas express a specific punctate immunostaining pattern for Cx43,

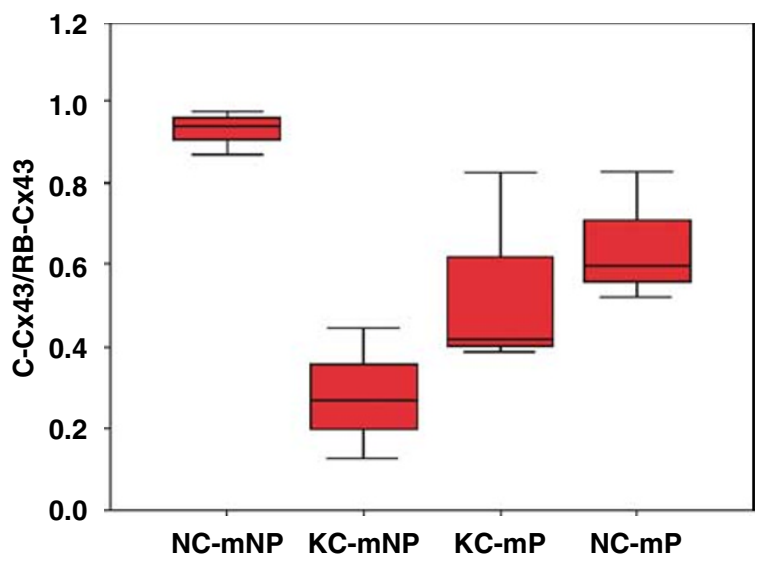

Figure 3 Densitometry analysis of Cx43 Western blotting, depicted in box plots. NC, normal cornea; KC, keratoconus cornea; $\mathrm{mNP}$, mean band intensity of nonphosphorylated $\mathrm{Cx} 43$; $\mathrm{mP}$, mean band intensity of phosphorylated Cx43; C-Cx43, corneal-Cx43 band intensity; RB-Cx43, rat brain- $\mathrm{Cx} 43$ band intensity. The $\mathrm{mNP}$ is 0.93 in normal corneas (SD: 0.052) and 0.349 in keratoconus corneas (SD: 0.190). The decrease is statistically significant $(P<0.05)$. The $\mathrm{mP}$ is also reduced in keratoconus corneas compared to normal corneas (0.548 with SD:0.172 and 0.604 with SD: 0.234 respectively). The difference is statistically insignificant though. 
located in the basal epithelial layer along the basic membrane, and in the anterior stroma. ${ }^{8,21}$ All normal human corneas examined displayed high concentrations of Cx43 with a characteristic immunolabeling pattern as described above. On the contrary, the immunostaining for $\mathrm{Cx} 43$ in six keratoconus corneas (corneas 2, 3, 4, 6, 7, and 8) was modified compared to normal corneas and this alteration was reflected in both the reduced signal intensity and the different immunolabelling pattern. The variety of Cx43 expression was confirmed with Western blotting analysis that showed four different intensity bands, each corresponding to the nonphosphorylated form and the three phosphorylated isoforms of $\mathrm{Cx} 43{ }^{22-24}$. Several studies have documented that dephosphorylation of $\mathrm{C} \times 43$ is associated with $\mathrm{C} \times 43$ gap junction degradation. ${ }^{10,25}$ The variability of $\mathrm{CX} 43$ expression may be explained by the fact that keratoconus pathophysiology displays a remarkable heterogeneity. ${ }^{1}$ Alternatively, changes in Cx43 expression may be a secondary effect of the disease and not the driving mechanism for the progression of the disease. This second explanation is supported by the fact that $\mathrm{Cx} 43$ appears normal in some of the pathological corneas studies.

An increasing number of studies associate various human diseases with defects in $\mathrm{C} \times 43$ gap junction activity, indicating that the role of $\mathrm{Cx} 43$ in many tissues is critical. Microarray studies have indicated that $\mathrm{C} \times 43$ expression is altered in both acute and chronic stages of multiple sclerosis, in Alzheimer's disease, Huntington's disease, and in other neural disorders. ${ }^{26-28}$ It has also been documented that oculo-dento-digital dysplasia, a syndrome characterized by developmental anomalies of the face, the eyes, the limbs, and the teeth, is caused by mutations in GjA1, which is the gene encoding $\mathrm{Cx} 43$. $^{29}$ Recently, it has also been reported that Axenfeld-Rieger syndrome, a disorder characterized by anterior segment dysgenesis, facial bone defects, and redundant periumbilical skin, is related to GJA1 gene mutations. ${ }^{30}$ However, there is no experimental evidence to link the locus of $C x 43$ gene to the keratoconus. On the other hand, familial keratoconus associated with cataract was mapped on the long arm of chromosome 15, however without any association to $\mathrm{C} \times 43$ gene, because the causative gene maps of the long arm of chromosome 15 has not been identified yet. ${ }^{31}$ The generation of knockout mice in which $C x 43$ gene expression is deficient has provided additional tools with which to dissect the role of Cx43 gap junctions in more detail. It has been demonstrated that $C x 43$ knock-out mice die perinatally due to an obstruction of the right outflow tract caused by cardiac malformations. ${ }^{32,33}$ Interestingly, knock out of $\mathrm{C} \times 43$ in the mouse produced an additional testicular defect presenting with outright failure of spermatogenesis. ${ }^{34}$ Finally, the $C x 43$-deficient mouse manifests numerous haematopoietic defects, which, along with the cardiac malformations, lead to death within hours of delivery. ${ }^{35}$ These data suggest that $\mathrm{C} \times 43$ gap junctions are an absolute requirement in a variety of diverse physiological processes, since defects in Cx43 gene are directly related to pathogenesis of disease.

Our results show that decrease in the total amount of Cx43 (NP-Cx43 and P-Cx43) along with a major downregulation of the active Cx43 isoforms (P-Cx43) may account for an important mechanism implicated in the keratoconus pathophysiology. However, further experiments are required to delineate whether specific pathways of $\mathrm{Cx} 43$ gap junction dysfunction occur in keratoconus and unravel their potential role in the development of the disease.

\section{References}

1 Rabinowitz YS. Keratoconus. Surv Ophthalmol 1998; 42(4): 297-319.

2 Kennedy RH, Bourne WM, Dyer JA. A 48-year clinical and epidemiologic study of keratoconus. Am J Ophthalmol 1986; 101: 267-273.

3 Kenney MC, Brown DJ, Rajeev B. Everett Kinsey lecture. The elusive causes of keratoconus: a working hypothesis. CLAO J 2000; 26(1): 10-13.

4 Meek KM, Tuft SJ, Huang Y, Gill PS, Hayes S, Newton RH et al. Changes in collagen orientation and distribution in keratoconus corneas. Invest Ophthalmol Vis Sci 2005; 46(6): 1948-1956.

5 Kumar NM, Gilula NB. The gap junction communication channel. Cell 1996; 84: 381-388.

6 Simon AM, Goodenough DA. Diverse functions of vertebrate gap junctions. Trends Cell Biol 1998; 8: 477-483.

7 Shibata Y, Kumai M, Nishii K, Nakamura K. Diversity and molecular anatomy of gap junctions. Med Electron Microsc 2001; 34: 153-159.

8 Dong Y, Roos M, Gruijters T, Donaldson P, Bullivant S, Beyer $\mathrm{E}$ et al. Differential expression of two gap junction proteins in corneal epithelium. Eur J Cell Biol 1994; 64: 95-100.

9 Spanakis SG, Petridou S, Masur SK. Functional gap junctions in corneal fibroblasts and myofibroblasts. Invest Ophthalmol Vis Sci 1998; 39: 1320-1328.

10 Lampe PD, Tenbroek EM, Burt JM, Kurata WE, Johnson RG, Lau AF. Phosphorylation of connexin 43 on serine 368 by protein kinase $\mathrm{C}$ regulates gap junctional communication. J Cell Biol 2000; 149(7): 1503-1512.

11 Solan JL, Lampe PD. Connexin phosphorylation as a regulatory event linked to gap junction channel assembly. Biochim Biophys Acta 2005; 1711(2): 154-163.

12 Nagase H, Woessner Jr JF. Matrix metalloproteinases. J Biol Chem 1999; 274(31): 21491-21494.

13 Brown D, Chwa MM, Opbroek A, Kenney MC. Keratoconus corneas. Increased gelatinase activity appears after modification of inhibitors. Curr Eye Res 1993; 12: 571-581.

14 Kenney MC, Chwa M, Opbroek AJ, Brown DJ. Increased gelatinolytic activity in keratoconus keratocyte cultures. A correlation to an altered matrix metalloproteinase-2/ tissue inhibitor of metalloproteinase ratio. Cornea 1994; 13: 114-124. 
15 Zhou L, Sawaguchi S, Twinning SS, Sugar J, Feder RS, Yue BY. Expression of degradative enzymes and protease inhibitors in corneas with keratoconus. Invest Ophthalmol Vis Sci 1998; 39(7): 1117-1124.

16 Sawaguchi S, Twining SS, Yue BY, Chang SH, Zhou X, Loushin $\mathrm{G}$ et al. Alpha 2-macroglobulin levels in normal human and keratoconus corneas. Invest Ophthalmol Vis Sci 1994; 35(12): 4008-4014.

17 Whitelock RB, Fukuchi T, Zhou L, Twining SS, Sugar J, Feder RS et al. Cathepsin G, acid phosphatase, and alpha 1-proteinase inhibitor messenger RNA levels in keratoconus corneas. Invest Ophthalmol Vis Sci 1997; 38(2): 529-534.

18 Maruyama Y, Wang X, Li Y, Sugar J, Yue BY. Involvement of $\mathrm{Sp} 1$ elements in the promoter activity of genes affected in keratoconus. Invest Ophthalmol Vis Sci 2001; 42(9): 1980-1985.

19 Alabain JH, Dossou H, Colin J, Floch HH. Levels of collagen degradation products (telopeptides) in the tear film of patients with keratoconus. Cornea 2000; 19(4): 474-476.

20 Matic M, Petrov IN, Chen S. Stem cells of the corneal epithelium lacks connexins and metabolite transfer capacity. Differentiation 1997; 61: 251-260.

21 Wolosin JM, Budak MT, Akinci MA. Ocular surface epithelial and stem cell development. Int J Dev Biol 2004; 48(8-9): 981-991.

22 Hao JL, Suzuki K, Lu Y, Hirano S, Fukuda K, Kumagai N et al. Inhibition of gap junction-mediated intercellular communication by TNF-a in cultured human corneal fibroblasts. Invest Ophthalmol Vis Sci 2005; 46(4): 1195-1200.

23 Anderson SC, Stone C, Tkach L, SundarRaj N. Rho and Rhokinase (ROCK) signalling in adherens and gap junction assembly in corneal epithelium. Invest Ophthalmol Vis Sci 2002; 43(4): 978-986.

24 Cruciani V, Mikalsen SO. Connexins, gap junctional intercellular communication and kinases. Biol Cell 2002; 94 433-443.

25 Saez JC, Retamal MA, Basilio D, Bukauskas FF, Bennett MVL. Connexin-based gap junctions hemichannels: gating mechanisms. Biochim Biophys Acta 2005; 1711: 215-224.
26 Lock C, Hermans G, Pedotti R, Brendolan A, Schadt E, Garren $\mathrm{H}$ et al. Gene-microarray analysis of multiple sclerosis lesions yields new targets validated in autoimmune encephalomyelitis. Nat Med 2002; 8: 500-508.

27 Aronica E, Gorter JA, Jansen GH, Leenstra S, Yankaya B, Troost D. Expression of connexin 43 and connexin 32 gap-junction proteins in epilepsy-associated brain tumors and in the perilesional epileptic cortex. Acta Neuropathol (Berlin) 2001; 101(5): 449-459.

28 Loring JF, Wen X, Lee JM, Seilhamer J, Somogyi R. A gene expression profile of Alzheimer's disease. DNA Cell Biol 2001; 20: 683-695.

29 Paznekas WA, Boyadjiev SA, Shapiro RE, Daniels O, Wollnik B, Keegan CE et al. Connexin 43 (GJA1) mutations cause a pleiotropic phenotype of oculodentodigital dysplasia. Am J Hum Genet 2003; 72: 408-418.

30 Cella W, de Vasconcellos JP, de Melo MB, Kneipp B, Costa FF, Longui CA et al. Structural assessment of PITX2, FOXC1, CYP1B1, and GJA1 genes in patients with Axenfeld-Rieger syndrome with developmental glaucoma. Invest Ophthalmol Vis Sci 2006; 47(5): 1803-1809.

31 Hughes AF, Dash DP, Jackson AJ, Frazer DG, Silvestri G. Familial keratoconus with cataract: linkage to the long arm of chromosome 15 and exclusion of candidate genes. Invest Ophth Vis Sci 2003; 44(12): 5063-5066.

32 Reaume AG, de Sousa PA, Kulliarni S, Langille BL, Zhu D, Davies TC et al. Cardiac malformation in neonatal mice lacking connexin 43. Science 1995; 267: 1831-1834.

33 Ya J, Erdtsieck-Ernste EBHW, de Boer PA, van Kempen MJ, Jongsma H, Moorman AF et al. Heart defects in Connexin43deficient mice. Circ Res 1998; 82: 360-366.

34 Risley MS, Tan IP, Farrell J. Gap junctions with varied permeability properties establish cell-type specific communication pathways in the rat seminiferous epithelium. Biol Reprod 2002; 67: 945-952.

35 Montecino-Rodriguez E, Leathers H, Dorshkind K. Expression of connexin 43 (Cx43) is critical for normal hematopoiesis. Blood 2000; 96: 917-924. 